\title{
ATIVIDADE FÍSICA HABITUAL EM ADULTOS COM DEFICIÊNCIA VISUAL: UMA REVISÃO SISTEMÁTICA
}

\author{
Roger Lima Scherer \\ Universidade Federal de Santa Catarina, Florianópolis, Santa Catarina, Brasil \\ Adair da Silva Lopes \\ Universidade Federal de Santa Catarina, Florianópolis, Santa Catarina, Brasil
}

\begin{abstract}
Resumo
O objetivo desta revisão foi identificar e avaliar as pesquisas publicadas envolvendo adultos com deficiência visual e a prática de atividade física. A busca foi realizada nas bases de dados: Scielo, Pubmed, Scopus, SportDiscus e ISI. Utilizou-se os operadores lógicos and, or e and not, para combinação dos descritores e termos utilizados para rastreamento das publicações. Apenas 07 artigos foram analisados na íntegra (quarta etapa: leitura completa e análise). Os resultados evidenciaram que, nos últimos 15 anos, poucos estudos foram publicados envolvendo a prática de atividades físicas em adultos com deficiência visual. Em sua maioria, os estudos já realizados envolveram amostras de crianças e idosos, além disso, há um direcionamento no campo médico e social com esta população.
\end{abstract}

Palavras-chave: Pessoas com Deficiência Visual. Atividade Motora. Revisão.

\section{Introdução}

$\mathrm{N}$

o Brasil, segundo o último censo (IBGE, 2000), há cerca de 24 milhões de pessoas com, pelo menos, uma deficiência, destes 16,6 milhões apresentaram deficiência visual e 150 mil são cegos.

Apesar de não haver dados oficiais, calcula-se que somente $10 \%$ das pessoas com deficiência no Brasil praticam algum tipo de atividade física regular (SOLER, 2005). De modo geral, os níveis de aptidão física entre os deficientes visuais tem se apresentado mais baixos quando comparados com pessoas sem deficiência visual (RURANGIRWA et al., 2006).

A atividade física e a aptidão física, segundo Nahas (2010), estão sendo associadas ao bem-estar, à saúde e à qualidade de vida de todas as pessoas, independente das faixas etárias. 
Os benefícios da atividade física estão bem estabelecidos e cada vez mais as pesquisas estão confirmando sua importância na manutenção da saúde e do bem estar. O exercício regular protege contra o aparecimento e a progressão de muitas doenças crônicas. Por isso, atividade física tem sido apontada como um componente importante de um estilo de vida saudável (ACMS, 2000).

O exercício físico pode ser utilizado como uma forma de lazer e, de restaurar a saúde dos efeitos que a rotina estressante de trabalho e dos estudos podem causar. Desta forma, é de vital importância que todas as pessoas se insiram em atividades físicas de forma regular (SILVA et al., 2010 ).

Pessoas com qualquer problema de natureza, física, mental ou sensorial tendem a ser menos ativas fisicamente, porém possuem as mesmas necessidades na prevenção de doenças e incremento da qualidade de vida. Atividades físicas regulares poderão reduzir sintomas de ansiedade e depressão, além de promover socialização e aumentar a percepção de bem-estar geral das pessoas com deficiência (NAHAS, 2010).

As pessoas com deficiência visual são mais propensas a engajarem-se em esportes e lazer passivos. As principais dificuldades para esta população praticarem atividades ativas são: limitações na aprendizagem tátil, inconveniência de se adaptar a novos ambientes rapidamente, funcionalidade e pressões econômicas (CHEN; LIN, 2011).

É necessário reconhecimento das limitações e das potencialidades destas pessoas neste processo de educação para uma vida ativa. Grande parte das pessoas com deficiência visual apresenta atraso motor nas atividades que envolvam equilíbrio, coordenação de movimentos, ritmo, esquema corporal, orientação espacial, além de hábitos posturais. Estas características evidenciadas, na maioria das pessoas com deficiência visual, são limitadores para a adesão de um estilo de vida ativo e uma boa percepção de qualidade de vida dos adultos com deficiência visual (MARQUES, 2005). Por isso, é necessário, com urgência, identificar que tipo de atividade física é eficaz para melhorar a qualidade de vida de pessoas com deficiência e propor intervenções para este fim (RIMMER et al., 2010) .

Em virtude do contexto atual das pessoas com deficiência visual, existe a necessidade de verificar o que está sendo estudado em relação a esta população, assim como, constatar quais pontos devem ser mais investigados futuramente. Sendo assim, o objetivo deste estudo foi re- 
alizar uma revisão sistemática da literatura sobre as pesquisas realizadas envolvendo a prática de atividade física em adultos (18-64 anos) com deficiência visual, nos últimos 15 anos.

\section{Procedimentos metodológicos}

Esta revisão sistemática da literatura foi realizada utilizando-se as bases de dados ("Scielo", "Pubmed", "Scopus", "SportDiscus" e "ISI"). As coletas foram realizadas nos domínios da Universidade Federal de Santa Catarina, em virtude do livre acesso aos periódicos do Portal Capes, de 20 a 26 de outubro de 2010. Os descritores utilizados foram definidos de acordo com as exigências de cada base de dados. Utilizou-se os operadores lógicos and, or e and not, para combinação dos descritores e termos utilizados para rastreamento das publicações.

A pesquisa no Scielo foi realizada em todos os índices, utilizando os seguintes descritores: "pessoa com deficiência visual" or "deficiente visual" and "aptidão física" or "atividade física" or "atividade motora". No Scopus por meio do título, resumo e palavras chaves, utilizando os descritores: "Visually Impaired Persons" OR "Visual impairment" AND "physical activity" OR "physical fitness" OR "Motor Activity". No Pubmed foram utilizados os descritores: ("Visually Impaired Persons" OR "Visual impairment") AND ("physical activity" OR "physical fitness" OR "Motor Activity"). No SportDiscus: ("Visually Impaired Persons" OR "Visual impairment") AND ("physical activity" OR "physical fitness" OR "Motor Activity"). Na base de dados ISI: (visually impaired persons OR visual impairment) and (physical activity OR physical fitness OR motor activity) and not (double-blind OR experimental OR randomized OR clinical trial). Desta forma, na presente revisão da literatura, utilizou-se para definir a população estudada as palavras: "pessoa com deficiência visual" e "deficiente visual" e para definir o desfecho da pesquisa as palavras: "aptidão física", "atividade física" e "atividade motora". $\mathrm{Na}$ base de dados da ISI, na qual foram encontrados muitos artigos além do objetivo definido para esta revisão, foi incorporado o operador lógico "and not" para as seguintes palavras: duplo cego, experimental, randomizado e ensaio clínico.

Para fazer parte do estudo os artigos deveriam atender aos seguintes critérios de inclusão: (a) amostra dos estudos deve ser somente de pessoas com deficiência visual; (b) artigos relacionados à atividade 
física; (c) artigos originais de pesquisa com seres humanos excluindo artigos de revisão; (d) faixa etária da amostra de 18 a 64 anos; (d) publicados de janeiro de 1995 a outubro de 2010. Não foram incluídos resumos, monografias, dissertações e teses decorrentes da dificuldade de realizar uma revisão sistemática nestas proporções.

Os estudos que preencheram os critérios de inclusão foram avaliados, independentemente do periódico. Quando finalizado o procedimento de busca, foram encontrados 07 artigos elegíveis para esta pesquisa de revisão sistemática.

A revisão foi realizada em quatro etapas (Figura 1). A primeira etapa consistiu da procura nas bases de dados utilizando-se os descritores selecionados. Ao finalizar esta etapa, foram encontrados 337 artigos. Porém, como houve consulta em cinco bases de dados, houve duplicidade de alguns estudos, finalizando com 313 artigos originais (Scielo: 06; Scopus: 23; Pubmed: 45; SportDiscus: 53 e ISI: 186) considerados elegíveis para a segunda etapa da pesquisa (análise dos títulos dos estudos). A segunda etapa finalizou com 81 pesquisas, sendo Scielo: 05; Scopus: 16; Pubmed: 11; SportDiscus: 34 e ISI: 15. A terceira etapa da pesquisa consistiu na análise dos resumos. Dentre os 81 artigos pré-selecionados, 08 atenderam a todos os critérios de inclusão: (Scopus: 04, Pubmed: 01, SportDiscus: 01 e ISI: 02).

A quarta etapa consistiu na leitura integral dos artigos. Nesta etapa ficaram somente 07 artigos, pois 01 artigo não possuía o texto na íntegra e não obtivemos resposta do autor quando solicitado. Os artigos foram excluídos, em sua maioria, por não apresentarem uma relação com a atividade física ou a pesquisa utilizar como amostra crianças ou idosos com deficiência visual.

Entre os artigos selecionados para análise na íntegra, foram observadas as seguintes etapas: amostra do estudo; objetivos definidos; aspectos éticos; conclusão da pesquisa. 


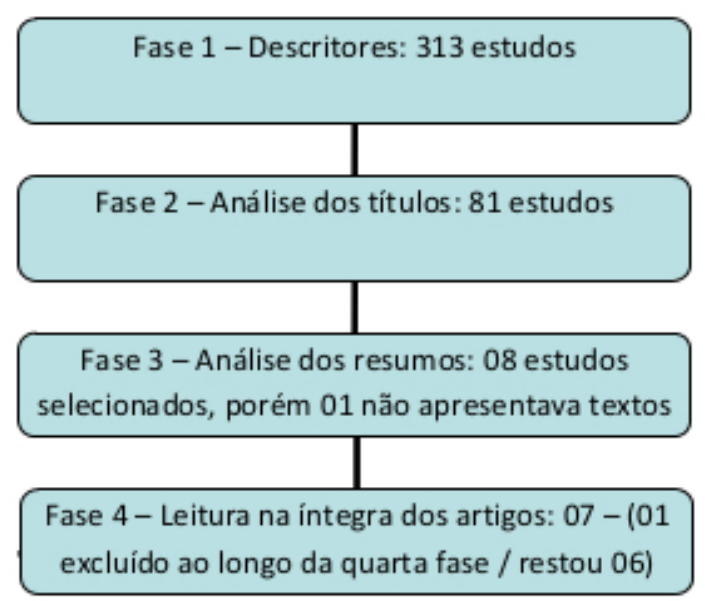

Figura 1: Organograma das etapas da revisão sistemática

\section{Resultados}

A tabela 1 apresenta os dados referentes às etapas da pesquisa e seus resultados. Dentre as 313 pesquisas encontradas, inicialmente, a base de dados da ISI foi o que apresentou um número maior de artigos ligado à população estudada com um desfecho vinculado a atividade física, encontrando 186 artigos, sendo aproximadamente 59\% da amostra total desta etapa. Porém a partir da segunda etapa, na qual foram analisados os títulos das pesquisas, verificou-se que a base de dados SportDiscus apresentou-se mais eficaz para o objetivo desta revisão, possuindo 34 artigos, aproximadamente $42 \%$ da amostra.

$\mathrm{Na}$ terceira etapa da pesquisa, foram analisados os resumos dos artigos. Dentre os 81 resumos analisados, foram selecionados 07 estudos para leitura na íntegra, sendo quatro da base de dados Scopus, um do Pubmed, um da SportDiscus e dois da ISI.

Os demais estudos não foram considerados elegíveis para a pesquisa (tabela 2), principalmente em virtude do critério faixa etária, possuindo a grande maioria dos estudos com referência a população de crianças, adolescentes ou idosos (aproximadamente 37\%).

Tabela 1: Resultado das etapas da revisão segundo os critérios estabelecidos em cada fase

\begin{tabular}{|c|c|c|c|c|c|c|c|c|c|c|c|}
\hline & \multicolumn{2}{|c|}{ Scielo } & \multicolumn{2}{|c|}{ Scopus } & \multicolumn{2}{|c|}{ Pubmed } & \multicolumn{2}{|c|}{ SportDiscus } & \multicolumn{2}{|c|}{ ISI } & \multirow[t]{2}{*}{ Total } \\
\hline & n & $\%$ & n & $\%$ & $\mathbf{n}$ & $\%$ & n & $\%$ & n & $\%$ & \\
\hline $1^{\circ}$ Etapa & 06 & 1,91 & 23 & 7,34 & 45 & 14,38 & 53 & 16,94 & 186 & 59,43 & 313 \\
\hline $2^{\circ}$ Etapa & 05 & 6,17 & 16 & 19,75 & 11 & 13,58 & 34 & 41,98 & 15 & 18,52 & 81 \\
\hline $3^{\circ}$ Etapa & & -- & 04 & 50,00 & 01 & 12,50 & 01 & 12,50 & 02 & 25,00 & 08 \\
\hline
\end{tabular}

Pensar a Prática, Goiânia, v. 16, n. 1, p. 1-319, jan./mar. 2013 
Além dos estudos excluídos decorrentes dos critérios pré-estabelecidos, uma pesquisa foi retirada do presente estudo em virtude de não apresentar texto completo em sua base de dados ou através do periódico capes, além do autor não responder a solicitação de envio do artigo para a pesquisa. Outro estudo foi excluído durante a leitura dos artigos na íntegra em virtude de sua amostra ser com idosos e a presente pesquisa apenas mencionar ao longo do estudo. Desta forma ficaram apenas seis estudos ao final desta revisão sistemática.

Tabela 2: Artigos inelegíveis para o estudo segundo os critérios estabelecidos

\begin{tabular}{|c|c|c|c|c|c|c|c|c|}
\hline $\begin{array}{l}\text { Critério de } \\
\text { exclusão }\end{array}$ & 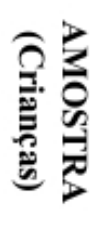 & 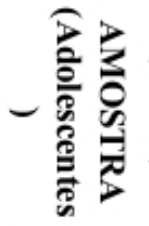 & 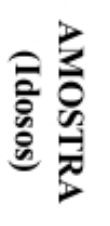 & 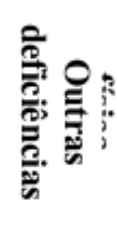 & 营: & 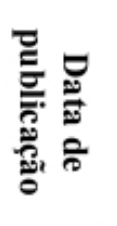 & 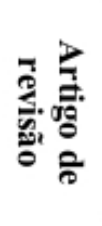 & 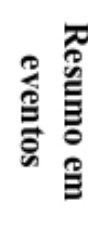 \\
\hline SCOPUS & 4 & 1 & 2 & & 1 & 2 & 1 & 1 \\
\hline PUBMED & 1 & & 4 & 2 & 2 & 1 & & \\
\hline SCIELO & & 1 & & & 2 & & 2 & \\
\hline$I S I$ & & 1 & 6 & 2 & 4 & & & \\
\hline SPORTDISCUS & 6 & 2 & 2 & 4 & 2 & 7 & 2 & 8 \\
\hline TOTAL & 11 & 5 & 14 & 8 & 11 & 10 & 5 & 9 \\
\hline
\end{tabular}

OBS: Não se encontrou um artigo da base de dados ISI na íntegra e o autor não respondeu a solicitação de envio.

No quadro 1 pode-se visualizar os seis estudos elegíveis para a quarta etapa (leitura integral do texto). 


\begin{tabular}{|c|c|c|c|c|}
\hline Titulo & Base & Autor/ano & Periódico & Principais conclusões \\
\hline $\begin{array}{l}\text { Effects of physical } \\
\text { inactivity on aortic } \\
\text { distensibility in } \\
\text { visually impaired } \\
\text { young men }\end{array}$ & Scopus & $\begin{array}{l}\text { Tetsuji Kakiyama; } \\
\text { Yasuko Koda; } \\
\text { Mitsuo Matsuda } \\
\text { (1999) }\end{array}$ & $\begin{array}{l}\text { European } \\
\text { Journal of } \\
\text { Applied } \\
\text { Physiology }\end{array}$ & $\begin{array}{c}\text { O aumento da atividade } \\
\text { fisica proporciona aos } \\
\text { individuos uma aorta } \\
\text { extensivel, assim como } \\
\text { naqueles individuos cegos } \\
\text { com diminuida e } \\
\text { desordenada atividade } \\
\text { física. }\end{array}$ \\
\hline $\begin{array}{l}\text { Kinetic Movement } \\
\text { Analysis in Adults } \\
\text { With Vision Loss }\end{array}$ & Pubmed & $\begin{array}{l}\text { Christopher Ray; } \\
\text { Michael Horvat; } \\
\text { Michael Williams; } \\
\text { Bruce Blasch } \\
\text { (2007) }\end{array}$ & $\begin{array}{l}\text { Adapted } \\
\text { Physical } \\
\text { Activity } \\
\text { Quarterly }\end{array}$ & $\begin{array}{l}\text { As conclusões dos autores } \\
\text { foram apoiadas por } \\
\text { trabalhos anteriores que } \\
\text { indica uma falta de resposta } \\
\text { adaptada à mudança no } \\
\text { movimento, incluindo o } \\
\text { cuidado, falta de vontade de } \\
\text { comprometer o seu centro } \\
\text { de gravidade, e uma falta de } \\
\text { força muscular para } \\
\text { individuos que são } \\
\text { deficientes visuais. }\end{array}$ \\
\hline $\begin{array}{c}\text { Bone mineral } \\
\text { density of visually } \\
\text { handicapped } \\
\text { women }\end{array}$ & Scopus & $\begin{array}{l}\text { Kirsti Uusi-Rasi; } \\
\text { Harri Sievänen; } \\
\text { Marjo Rinne; } \\
\text { Pekka Oja; Ilkka } \\
\text { Vuori (2001) }\end{array}$ & $\begin{array}{c}\text { Clinical } \\
\text { Physiology }\end{array}$ & $\begin{array}{l}\text { A deficiência visual, sem } \\
\text { condições concomitantes } \\
\text { que afetam o metabolismo } \\
\text { ósseo parecem ser um risco } \\
\text { para baixa densidade } \\
\text { mineral óssea. }\end{array}$ \\
\hline $\begin{array}{l}\text { The Value of } \\
\text { Physical Fitness for } \\
\text { a Young Man who } \\
\text { is Visually } \\
\text { Impaired with } \\
\text { Multiple Medical } \\
\text { Disorders }\end{array}$ & Scopus & $\begin{array}{l}\text { Marley W.P.; } \\
\text { Beverly-Mullins } \\
\text { J.M. (1997) }\end{array}$ & $\begin{array}{l}\text { Journal of } \\
\text { Visual } \\
\text { Impairment \& } \\
\text { Blindness }\end{array}$ & $\begin{array}{l}\text { Os ganhos obtidos pelo } \\
\text { jovem permitiu um melhor } \\
\text { controle das suas limitações } \\
\text { visuais e dos múltiplos } \\
\text { transtornos clínicos, que, } \\
\text { em contrapartida, } \\
\text { proporcionou uma melhor } \\
\text { independência em seu } \\
\text { cotidiano. }\end{array}$ \\
\hline $\begin{array}{l}\text { Mental Imagery in } \\
\text { Athletes with } \\
\text { Visual Impairments }\end{array}$ & SportDiscus & $\begin{array}{l}\text { Kate A. T. Eddy; } \\
\text { Stephen D. } \\
\text { Mellalieu (2003) }\end{array}$ & $\begin{array}{l}\text { Adapted } \\
\text { Physical } \\
\text { Activity } \\
\text { Quarterly }\end{array}$ & $\begin{array}{c}\text { A imagem mental tem } \\
\text { emergido como uma } \\
\text { habilidade psicológica útil } \\
\text { para os atletas que são } \\
\text { deficientes visuais. E para } \\
\text { obter o maior beneficio } \\
\text { desta habilidade, há uma } \\
\text { necessidade destas imagens } \\
\text { mentais se tornarem um } \\
\text { processo mais consciente } \\
\text { para os atletas. }\end{array}$ \\
\hline $\begin{array}{l}\text { Quality of Life and } \\
\text { Visual Function in } \\
\text { Patients with } \\
\text { Intermediate } \\
\text { Uveitis }\end{array}$ & ISI & $\begin{array}{l}\text { C. C. Murphy; E. } \\
\text { H. Hughes; N. A. } \\
\text { Frost; A. D. Dick } \\
\text { (2005) }\end{array}$ & $\begin{array}{c}\text { British Journal } \\
\text { of } \\
\text { Ophthalmology }\end{array}$ & $\begin{array}{l}\text { O impacto da qualidade da } \\
\text { visão prejudicada está } \\
\text { relacionado à saúde em } \\
\text { geral e na qualidade de vida } \\
\text { dos pacientes com uveíte } \\
\text { intermediária. }\end{array}$ \\
\hline
\end{tabular}

Quadro 1: Artigos elegíveis para o estudo segundo os critérios estabelecidos 
A amostra dos estudos para a presente pesquisa foi delimitada para adultos com deficiência visual, porém há uma diversidade com relação às funções, possuírem ou não patologias, ou serem atletas ou não. Dos seis estudos, apenas um havia pacientes com alguma patologia instaurada, os demais estudos eram com pessoas com a deficiência visual, alguns com atletas outros com pessoas ativas ou sedentárias fisicamente.

Os estudos apresentaram objetivos diversos com relação à pessoa com deficiência visual e seu vínculo com a atividade física. Ray, et al. (2007) buscaram avaliar a capacidade de movimento das pessoas com deficiência visual e comparar aquelas pessoas sem deficiência visual; Uusi-Rasi et al. (2001) verificaram se há diferenças na densidade mineral óssea de mulheres com e sem deficiência visual; Kakiyama, Koda e Matsuda (1999) verificaram se há um aumento na rigidez aórtica com o progresso da deficiência visual; Marley e Beverly-Mullins (1997) verificaram o valor de intervenção de um ano em um rapaz com deficiência visual com múltiplos transtornos clínicos através de aconselhamento nutricional e educação com relação a diabetes, além de um programa de exercício físico; Eddy e Mellalieu (2003) investigaram o uso de imagens mentais em atletas com deficiência visual; e Murphy et al. (2005) verificaram a qualidade de vida e visão funcional de pacientes com uveítes intermediárias.

Com relação aos aspectos éticos das pesquisas com seres humanos, três estudos informaram ao longo do texto a aprovação do comitê de ética, assim como o consentimento livre esclarecido dos voluntários, porém os outros três estudos nada mencionam sobre este aspecto.

\section{Discussão}

Dentre as bases de dados, a SportDiscus e a Scopus foram as que apresentaram os maiores números de pesquisas, porém com os critérios pré-estabelecidos e, principalmente, a delimitação quanto a faixa etária, restringiu muito o número de estudos na presente pesquisa.

Os estudos elegíveis foram variados, porém, apenas um envolveu a intervenção de um programa de exercício físico destinado aos adultos com defíciência visual. Não se verificou nesta pesquisa uma tendência para algum tipo de estudo específico ou amostra preferida como foco de pesquisa, mostrando claramente a recente área de pesquisa e o quanto deve ser explorada em suas diversas áreas de atuação (biome- 
cânica, cineantropometria, atividade física relacionada à qualidade e estilo de vida, psicologia do esporte, pedagogia do esporte, nutrição esportiva, entre outros).

Os estudos que não apresentaram aspectos éticos ligados à pesquisa, dois deles foram publicados na década de 90 e o estudo de 2003 não mencionou nada sobre os preceitos éticos, porém descreve ao longo sobre o estudo piloto realizado anteriormente e a pesquisa é qualitativa, utilizada para explorar as vivências dos atletas no processamento e uso de imagens mentais.

Uusi-Rasi et al. (2001) mencionaram que a deficiência visual, sem condições concomitantes que afetam o metabolismo ósseo parecem ser um risco para baixa densidade mineral óssea. A magnitude do déficit da densidade mineral óssea observada nas mulheres de meia-idade da pesquisa com a deficiência visual, sugere que o risco de fraturas relacionadas a defeitos visuais pode ser aumentado. Porém, os autores mencionaram que o estudo é transversal, e o tamanho da amostra é pequena, dados epidemiológicos ou prospectivos são necessários para confirmar a deficiência visual como um fator de risco para osteoporose.

Para Ray, et al. (2007) os indivíduos com perda de visão normalmente são mais cuidadosos e relutantes ao utilizar os movimentos que comprometem seu centro de gravidade. Esta pode ser uma resposta adaptativa para compensar a perda da visão ou baixo condicionamento físico. As conclusões dos autores foram apoiadas por trabalhos anteriores que indica uma falta de resposta adaptada à mudança no movimento, incluindo o cuidado, falta de vontade de comprometer o seu centro de gravidade e uma falta de força muscular para indivíduos que são deficientes visuais. Como as tarefas escolhidas no estudo representaram vários componentes do funcionamento (flexibilidade, força, equilíbrio e controle motor), os autores encontraram certa difículdade em conclusões com mais consistência sobre os componentes da aptidão física analisados. Desta forma, os achados foram relativos ao desempenho da tarefa global e há necessidade de estudos futuros para explorar os diferentes componentes. Além disso, intervenções focadas no aumento da mobilidade e a capacidade para responder adequadamente às mudanças na estabilidade e iniciar o movimento entre os deficientes visuais devem ser examinadas. A atividade física deve ser incentivada a desenvolver e manter a força muscular necessária para a realização de tarefas funcionais, auto-suficiência e independência. 
No estudo de Kakiyama, Koda e Matsuda (1999), os resultados foram consistentes com a idéia de que a inatividade física excessiva diminui a rigidez da aorta. Os principais determinantes das propriedades mecânicas da parede arterial são elastina e colágeno. Estudos anteriores têm sugerido que o exercício físico pode retardar a progressão da degeneração associada à idade da aorta e que esse efeito provavelmente foi responsável na produção de uma distensão na parede da aorta. A hipótese dos autores é que a degeneração de elastina seria acelerada por uma atividade física desordenada. Algumas hipóteses são reportadas com relação a patologias hereditárias ou sobre as paredes da musculatura lisa vascular dos indivíduos cegos, entre outros para uma possível causa para esta disistabilidade aórtica. Os autores sugerem novas investigações na busca de aspectos diferenciados para verificar as possíveis causas desse fenômeno. Concluem que o aumento da atividade física proporciona aos indivíduos uma aorta extensível, assim como naqueles indivíduos cegos com diminuida e desordenada atividade física.

No estudo referente a um programa de intervenção em um indivíduo com problemas de saúde associados como diabetes tipo I, hipertensão e um disturbio lipídico, Marley e Beverly-Mullins (1997) encontraram o valor do exercício estruturado e relacionados a estratégias pró-ativas para motivação de uma pessoa cega com vários problemas de saúde. Os ganhos obtidos pelo jovem permitiu um melhor controle das suas limitações visuais e dos múltiplos transtornos clínicos, que, em contrapartida, proporcionou uma melhor independência em seu cotidiano. No entanto, é pouco provável que ele ou pessoas com condições semelhantes poderiam realizar esse programa sozinho. Uma regressão após sua saída do programa supervisionado reforça a necessidade de apoio continuado por outros profissionais da saúde e de profissionais de reabilitação para pessoas cegas.

Eddy e Mellalieu (2003) estudaram a utilização de imagens mentais em atletas de goalball. Os resultados sugerem que atletas com deficiências visuais utilizam predominantemente o processo de imagens mentais a partir de uma perspectiva interna. Os canais para entrada para essa imagem parecem ser derivados de todas as modalidades sensoriais do indivíduo, incluindo as formas cinestésica, espacial, tátil, visual e auditiva. Além disso, os atletas também relataram o uso de ambas as formas cognitivas e motivacionais de imagens mentais para atingir resultados psicológicos em uma variedade de contextos de for- 
mação e competição. As implicações práticas desta investigação sugerem que a imagem mental tem emergido como uma habilidade psicológica útil para os atletas que são deficientes visuais. E para obter o maior benefício desta habilidade, há uma necessidade destas imagens mentais se tornarem um processo mais consciente para os atletas, pois não aparecem atualmente para ser usado em todo seu potencial neste grupo estudado.

Murphy et al. (2005) estudaram a qualidade de vida e a função visual em pacientes com uveíte intermediária. Este inquérito foi realizado por meio do questionário SF-36, no qual possui 36 itens em oito dimensões. Os itens são relacionados com a capacidade funcional; aos aspectos físicos; sobre a dor; sobre o estado geral de saúde; sobre a vitalidade; sobre os aspectos sociais; aspectos emocionais e relacionados com a saúde mental. Para função visual foram realizados alguns testes para verificar os níveis de função visual assim como exames clínicos. Os autores encontraram que a função visual e a saúde em geral estão bem conservadas na maioria dos pacientes com uveíte intermediária. Além disso, destacam que o impacto da qualidade da visão prejudicada está relacionado à saúde em geral e na qualidade de vida dos pacientes com uveíte intermediária.

Ao longo destes quinze anos, poucas pesquisas foram publicadas, nas bases de dados pesquisadas, envolvendo adultos com deficiência visual e a atividade física. A maioria dos estudos foi realizada em outras faixas etárias, principalmente com crianças e idosos. Além disso, ainda há um direcionamento muito grande das pesquisas envolvendo pessoas com deficiência visual para o campo médico e social, apresentando poucos estudos direcionados à qualidade de vida/estilo de vida ou programas de intervenção mais direcionados a esta população. O campo de pesquisa é extremamente amplo e rico na alteridade destes indivíduos. Portanto, constatou-se a pesquisa envolvendo a prática de atividades físicas para pessoas com deficiência visual é uma área de pesquisa que precisa ser explorada para preencher as lacunas do conhecimento acadêmico-científico.

Physical activity in the adults with visual impairment: a systematic review

\begin{abstract}
The aim of this review was to identify and evaluate published researches involving adults with visual impairment and physical activity. The search was conducted in databases: Scielo, Pubmed, Scopus, SportDiscus and ISI. Descriptors were used in
\end{abstract}


Portuguese and their equivalents in English, using logical operators. Only 07 articles were analyzed in full. (fourth step: complete reading and analysis). The results showed that in the last 15 years, few studies have been published involving the practice of physical activity in adults with visual impairment, in most studies mainly with children and the elderly. Moreover, there is still a very big direction in the medical and social fields with this population.

Keywords: Visually Impaired Persons. Motor Activity. Review.

La actividad física habitual en adultos con deficiencia visual: una revisión sistemática

\section{Resumen}

El objetivo de esta revisión fue identificar y evaluar las investigaciones publicadas con adultos con discapacidad visual y la actividad física. La búsqueda se realizó enbases de datos: Scielo, Pubmed, Scopus y Sportdiscus ISI. Se utilizaron losoperadores lógicos AND, OR y AND NOT para la combinación de descriptores y términos utilizados para rastrear las publicaciones. Sólo 07 artículos fueron analizados en su totalidad (Cuarto paso: lectura y análisis a fondo). Los resultados mostraron que en los últimos 15 años, pocos estudios se han publicado que implica la actividad física en los adultos con impedimentos visuales. La mayoría de los estudios previos implicados muestras de los niños y los ancianos, además, hay una dirección en el ámbito médico y social con esta población.

Palabras clave: Personas con Daño Visual. Actividad Motora. Revisión.

\section{Referências}

ACMS - American College of Sports Medicine. Teste de Esforço e Prescrição de Exercício. Rio de Janeiro: REVINTER, 2000.

CHEN, C. C.; LIN, S. Y. The impact of rope jumping exercise on physical fitness of visually impaired students. Research in Developmental Disabilities, v. 32, p.25-29, 2011.

EDDY, K. A. T.; MELLALIEU, S. D. Mental Imagery in Athletes with Visual Impairments. Adapted Physical Activity Quarterly, v. 20, n. 4, p. 347-368, out., 2003.

IBGE. Censo Demográfico 2000: Características gerais da população: resultados da amostra. Rio de Janeiro: Instituto Brasileiro de Geografia e Estatística - IBGE, 2000.

KAKIYAMA, T.; KODA, Y.; MATSUDA, M. Effects of physical inactivity on aortic distensibility in visually impaired young men. Eu- 
ropean Journal of Applied Physiology, v. 79, n. 3, p. 205-211, fev., 1999.

MARLEY, W. P. BEVERLY-MULLINS, J. M. The Value of Physical Fitness for a Young Man who is Visually Impaired with Multiple Medical Disorders. Journal of Visual Impairment \& Blindness, v. 91, n. 4, p. 341-346, 1997.

MARQUES, A. C. O papel da atividade física na saúde e qualidade de vida de pessoas deficientes. In: Congresso Brasileiro de Atividade Física \& Saúde, 5., 2005, Florianópolis. Anais... Florianópolis: UFSC, 2005. p. 17.

MURPHY, C. C. et al. Quality of life and visual function in patients with intermediate uveitis. British Journal of Ophthalmology, v. 89, n. 9, p. 1161-1165, set., 2005.

NAHAS, M. V. Atividade Física, Saúde e Qualidade de Vida: Conceitos e Sugestões para um Estilo de Vida Ativo. 5. ed. Rev. e Atual., Londrina: Midiograf, 2010.

RAY, C. et al. Kinetic Movement Analysis in Adults With Vision Loss. Adapted Physical Activity Quarterly, v. 24, n. 3, p. 209-217, jul., 2007.

RIMMER, J. H. et al. Accessibility of Health Clubs for People With Mobility Disabilities and Visual Impairments. American Journal of Public Health, v. 95, n. 11, p. 2022-2028, nov., 2005.

RURANGIRWA, J. et al. Healthy behaviors and lifestyles in young adults with a history of developmental disabilities. Research in Developmental Disabilities, v. 27, n. 4, p. 381-399, jul./ago., 2006.

SILVA, R. S. et al. Atividade Física e Qualidade de Vida. Ciência \& Saúde Coletiva, Rio de Janeiro, v. 15, n. 1, p. 115-120, jan., 2010.

SOLER, R. Educação física inclusiva na escola em busca de uma escola plural. Rio de Janeiro: Sprint, 2005. 254 p.

UUSI-RASI, K. et al. Bone mineral density of visually handicapped women. Clinical Physiology, v. 21, n. 4, p. 498-503, 2001. 
DOI 10.5216/rpp.v16i1.17356

Recebido em: 29/02/2012

Revisado em: 22/05/2012

Aprovado em: 03/07/2012

\section{Endereço para correspondência}

adair@cds.ufsc.br

Adair da Silva Lopes

Universidade Federal de Santa Catarina

Centro de Desportos, Departamento de Educação Física.

Campus Universitário - Trindade

Trindade

88040-900 - Florianopolis, SC - Brasil 